\title{
E D I T O R I A L
}

\section{Breathing New Life into Interstitial Lung Disease in Rheumatoid Arthritis}

\author{
Peter K. Gregersen, M.D., and Ellen M. Gravallese, M.D.
}

Clinical heterogeneity is a hallmark of many autoimmune disorders, and clinical or subclinical pulmonary involvement is a common extraarticular feature of the rheumatoid arthritis (RA) phenotype. High-resolution computed tomography reveals evidence of pulmonary abnormalities in more than half of patients with RA, and clinically significant interstitial lung disease (ILD) will develop in approximately $10 \%$ of patients. ${ }^{1}$ It is currently difficult to identify these patients and to intervene early in the clinical course of their lung disease.

The application of genomewide association studies to the search for common genetic variants involved in autoimmune and inflammatory diseases has produced a rich harvest of hundreds of genetic associations. However, most of these associations have low effect sizes, and practical implications for understanding, diagnosing, and treating these disorders have been extremely slow to emerge. In the case of RA, the effect of HLA genes dominates over the other 100 confirmed genetic associations. ${ }^{2}$ Although HLA genes control some of the immunologic features of the disease, the effect of HLA on other clinical features, including lung disease, is modest. In fact, no clear subsets of disease have been identified from consideration of other genetic variants. The report by Juge et al. now appearing in the Journal provides an exciting exception to this trend. ${ }^{3}$

Genetic studies involving people with idiopathic pulmonary fibrosis have implicated a number of common and rare genetic variants in disease pathogenesis, the strongest of which is a gain-of-function promoter variant in MUC5B $(\text { rs } 35705950)^{4}$; this accounts for approximately
30 to $35 \%$ of the genetic risk of the development of idiopathic pulmonary fibrosis. Strikingly, in the current study by Juge et al. involving patients with RA, those who carried this variant had a higher risk of ILD than those who did not, with an odds ratio of 3.1 ( $95 \%$ confidence interval, 1.8 to 5.4), even when the researchers took into account smoking (a known risk factor for both ILD and RA), age, and sex. This promoter variant does not confer risk of interstitial pneumonia among patients with systemic sclerosis. ${ }^{5}$ The increased risk that was associated with the variant was found across multiple ethnic groups (with the exception of Asians, for whom the sample sizes and the rarity of the risk allele limited statistical power), a finding that adds to the likelihood that this is indeed a causative variant. Several other common variants that have previously been associated with the risk of ILD did not show this effect in the context of RA, although the sample sizes in the current study do not provide the power to test for these more modest associations.

The pathogenesis of idiopathic pulmonary fibrosis almost certainly involves several mechanisms, including abnormalities in surfactant secretion leading to alveolar damage, predisposition to cell senescence associated with telomere shortening, and interference with ciliary clearance. Mucins are glycosylated proteins that represent an important component of the mucus that adheres to ciliated epithelium in the lung, providing a defensive barrier, as well as a clearance mechanism for inhaled particles and pathogens. As Juge and colleagues show, the MUC5B protein is expressed in bronchiolar epithelium and in 
metaplastic epithelia lining the honeycomb cysts typical of RA-associated ILD. Genetically driven overexpression of MUC5B may contribute to overproduction of MUC5B protein, with subsequent interference in ciliary clearance, or disrupt normal lung-repair mechanisms. ${ }^{6}$

Paradoxically, in addition to being associated with an elevated level of expression and risk of disease, the MUC5B promoter risk allele is also associated with an improved outcome in patients with idiopathic pulmonary fibrosis. ${ }^{7}$ The prevalence of this allele in many populations suggests that it may be under selection pressure, perhaps driven by improved resistance to infection or enhanced tissue repair. A late-onset illness such as ILD is highly unlikely to be a major selective disadvantage. ${ }^{6}$ In the event that an improved outcome of lung disease in patients with RA with the MUC5B risk allele is observed, one might hypothesize that other patients with RA with ILD but who lack the MUC5B risk allele carry more rare risk alleles in other genes acting in different pathways.

Fujii et al. have previously reported that shortened telomeres are a feature of patients with $\mathrm{RA},{ }^{8}$ and there is evidence for accelerated telomere shortening in patients with ILD who carry risk alleles in this pathway. Moreover, in a recent small study, persons with RA and ILD who carried rare risk alleles in genes encoding proteins that regulate telomere length (TERT, RTEL1, and PARN) also exhibited telomere shortening. ${ }^{9} \mathrm{Al}-$ though there are many intriguing possibilities to explain shortened telomeres in patients with RA, the association of RA with ILD may be a partial explanation. Genes in the telomere pathway were not implicated in the genomewide association studies of RA. ${ }^{10}$ Because the genotyping chips used in genomewide association do not detect rare variants, it remains possible that rare variants in this pathway cause susceptibility to RA, with or without concomitant ILD. In addition, common variants, such as MUC5B, may yet show up in future genomewide association studies of RA using updated genotyping platforms that better capture common variation at certain loci.

The strong association of the MUC5B promoter variant with ILD in patients with RA raises the question of whether all patients with RA should be evaluated for MUC5B carrier status. This will be most appropriate when early detection can mandate preventive or effective therapies. On the other hand, once clinical disease is present, MUC5B genotyping might provide information on prognosis and, as understanding of pathogenesis improves, may guide management. Overall, the results of Juge et al. support the concept that genetics really can add to our understanding of disease heterogeneity, and the results provide hope that this approach will lead to further insights in the future.

Disclosure forms provided by the authors are available with the full text of this editorial at NEJM.org.

From the Feinstein Institute for Medical Research, Robert S. Boas Center for Genomics and Human Genetics, Manhasset, NY (P.K.G.); and the Department of Medicine, Division of Rheumatology, University of Massachusetts Medical School, Worcester (E.M.G.).

This editorial was published on October 20, 2018, at NEJM.org.

1. Olson AL, Swigris JJ, Sprunger DB, et al. Rheumatoid arthritisinterstitial lung disease-associated mortality. Am J Respir Crit Care Med 2011;183:372-8.

2. van Drongelen V, Holoshitz J. Human leukocyte antigendisease associations in rheumatoid arthritis. Rheum Dis Clin North Am 2017;43:363-76.

3. Juge P-A, Lee JS, Ebstein E, et al. MUC5B promoter variant and rheumatoid arthritis with interstitial lung disease. $\mathrm{N}$ Engl $\mathrm{J}$ Med. DOI: 10.1056/NEJMoa1801562.

4. Seibold MA, Wise AL, Speer MC, et al. A common MUC5B promoter polymorphism and pulmonary fibrosis. N Engl J Med 2011;364:1503-12.

5. Peljto AL, Steele MP, Fingerlin TE, et al. The pulmonary fibrosis-associated MUC5B promoter polymorphism does not influence the development of interstitial pneumonia in systemic sclerosis. Chest 2012;142:1584-8.

6. Evans CM, Fingerlin TE, Schwarz MI, et al. Idiopathic pulmonary fibrosis: a genetic disease that involves mucociliary dysfunction of the peripheral airways. Physiol Rev 2016;96:1567-91. 7. Peljto AL, Zhang Y, Fingerlin TE, et al. Association between the MUC5B promoter polymorphism and survival in patients with idiopathic pulmonary fibrosis. JAMA 2013;309:2232-9.

8. Fujii H, Shao L, Colmegna I, Goronzy JJ, Weyand CM. Telomerase insufficiency in rheumatoid arthritis. Proc Natl Acad Sci U S A 2009;106:4360-5.

9. Juge PA, Borie R, Kannengiesser C, et al. Shared genetic predisposition in rheumatoid arthritis-interstitial lung disease and familial pulmonary fibrosis. Eur Respir J 2017;49(5):pii:1602314. 10. Okada Y, Wu D, Trynka G, et al. Genetics of rheumatoid arthritis contributes to biology and drug discovery. Nature 2014; 506:376-81.

DOI: 10.1056/NEJMe1811767

Copyright (c) 2018 Massachusetts Medical Society. 